\title{
Waist Structure Design and Analysis of Joint Type Robot
}

\author{
Wei Wu \\ Jiangxi Technical College of Manufacturing, Department of Mechanical Engineering
}

\author{
Keywords: Joint Type; Robot; Structure; Trajectory Planning
}

\begin{abstract}
The development of industrial technology makes the application field of robot become wider. Joint type robot research and development and trajectory planning research have got more concerns. Joint type industrial robots are famous for their characteristics of big scope of work, flexible motion, and compact structure and so on, which are popular with robot's designers and users. This paper mainly introduces the mechanical structure design, kinematics analysis and control system design of series joint type robots, and makes detailed analysis and design with the example of five degrees of freedom robot joints in mechanical structure, kinematics analysis, the structure of hardware driver, software control system, servo communication protocols and so on.
\end{abstract}

\section{Introduction}

Joint type robot attracts the attention of designers and users with wide range of work, flexible motion, compact structure, grabbing the object near the base and other characteristics. This paper makes study the analysis for six degree of freedom joint type robot arm part, mainly including the overall plan design, kinematics analysis of the selected robot, trajectory planning and workspace analysis, as well as the virtual prototype design and kinematics simulation. This topic designs three degrees of freedom robot arm, which sets the stage for the subsequent subject; the six degree of freedom robot can be used for teaching and scientific research after design, also for further research and development, so the research of this topic has practical significance and wide application prospect.

In order to meet the requirements of robot operation content, this paper has carried on the research of relevant key technologies. At first, the author puts forward several schemes, and after analysis and comparison, using Pro/Engineer and AutoCAD software model for robot structure model design after selecting the most optimal solution; Secondly, the paper performs kinematics analysis, using D-H method to establish the coordinate transformation matrix, calculating the positive and inverse solution of equations of motion, using $\mathrm{VC}++$ to make the solution of the positive and inverse kinematics interface; Finally, use ADAMS software to simulate the robot arm movement, and the results are analyzed, virtual prototype technology in mechanical design is tried accumulating experience.

\section{The Characteristics of the Robot Mechanical Design}

Unique structural characteristics of robot can be roughly classified into the following four points. Joint type industrial robot manipulator can be simplified into open type linkage with the connecting head-tai rod and open end. In order to achieve the coordinate motion, in the most of the time, the end of the linkage is unable to support, thus the structure of the manipulator rigidity is poor, and changes along with the connecting rod posture the space.

In the open type of linkage that forms the manipulator, each rod has independent drive, and thus it belongs to the active linkage. Different with the common linkage, in the linkage, all the connecting rod movement are from the same driving source, and the motion of each connecting rod is restricted. As the movement of each connecting rod is independent, different connecting rod movement has no compliance relationship, so the movement of manipulator is more flexible.

The transient process of connecting rod drive torque has very complex change in time domain 
and is related to the feedback signal of executive component. The connecting rod drive belongs to servo control type, and thus it has higher requirements for the stiffness, clearance and motion precision of mechanical transmission system. The three joint drivers used in this paper are stepper motor driver, belongs to open loop control.

The stress state, stiffness conditions and dynamic performance of linkage change along with the pose, therefore, it's very prone to vibration or other unstable phenomenon.

\section{Robot Position and Pose Description}

In order to describe the movement relationship among the connecting rods of robots, robots and environment (artifacts and obstacles), it's usually studies as a rigid body. In a right angle $\{\mathrm{A}\}$ (Descartes) coordinate system, any point $\mathrm{P}$ in the space position can be expressed in the column vector ${ }^{A} P$ of $3 \times 1$ :

$$
{ }^{A} P=\left[\begin{array}{c}
P_{x} \\
P_{y} \\
P_{z}
\end{array}\right]
$$

$P_{x}, P_{y}, P_{z}$ are the coordinate components of $\mathrm{P}$ in coordinate $\{\mathrm{A}\}$.

To determine the orientation (pose) of a rigid body in coordinate system $\{\mathrm{A}\}$, build a right angle coordinate system $\{\mathrm{B}\}$ to join with this rigid body, then use three main vector ${ }^{A} X_{B},{ }^{A} Y_{b},{ }^{A} Z_{B}$ of coordinate $\{\mathrm{B}\}$ relating to the direction cosine of coordinate system $\{\mathrm{A}\}$ to form $3 \times 3$ matrix:

$$
{ }^{A} R_{B}=\left[{ }^{A} X_{B},{ }^{A} Y_{B},{ }^{A} Z_{B}\right]
$$

or

$$
{ }^{A} R_{B}=\left[\begin{array}{lll}
R_{11} & R_{12} & R_{13} \\
R_{21} & R_{22} & R_{23} \\
R_{31} & R_{32} & R_{33}
\end{array}\right]
$$

To express the pose of the rigid body relative to coordinate system $\{\mathrm{A}\},{ }^{A} R^{B}$ is called rotation matrix, and superscript represents reference coordinate system $\{\mathrm{A}\}$, and subscript represents the described coordinate system $\{\mathrm{B}\}$. There are 9 elements in the rotation matrix. The first column represents the cosine values generated by $\mathrm{x}$ axis with $\mathrm{x}, \mathrm{y}, \mathrm{z}$ axis in coordinate system $\{\mathrm{A}\}$ respectively. The $2^{\text {nd }}$ and $3^{\text {rd }}$ columns represent the cosine values generated by $y, z$ axis in coordinate system $\{B\}$ with $x, y$, $\mathrm{Z}$ axis in coordinate system $\{\mathrm{A}\}$ respectively.

If a rigid body has certain angle in the orientation of a coordinate system with the axis, we can use rotation matrix to express it. From the geometric meaning, the rigid body can be considered as reaching current direction after rotating certain angle with a coordinate system. The rotation matrix with $\mathrm{x}, \mathrm{y}, \mathrm{z}$ axes are as follows:

$$
R(x, \theta)=\left[\begin{array}{ccc}
1 & 0 & 0 \\
0 & \cos \theta & -\sin \theta \\
0 & \sin \theta & \cos \theta
\end{array}\right]
$$




$$
\begin{aligned}
& R(y, \theta)=\left[\begin{array}{ccc}
\cos \theta & 0 & \sin \theta \\
0 & 1 & 0 \\
-\sin \theta & 0 & \cos \theta
\end{array}\right] \\
& R(x, \theta)=\left[\begin{array}{ccc}
\cos \theta & -\sin \theta & 0 \\
\sin \theta & \cos \theta & 0 \\
0 & 0 & 1
\end{array}\right]
\end{aligned}
$$

We can see that the point position can be expressed with position vector; the orientation can be expressed with rotation matrix, as the formula above. For several rotations around each axis, just multiply the basic rotation matrixes.

\section{Comparison Analysis and Selection of Robot Driver Scheme}

There are 4 kinds of robot driver's modes:

Stepper motor driver; Stepper motor is a kind of electromagnetic actuator of incremental motion, and this element is a device converting digital pulse to rotation or linear increment motion. When using the appropriate control, the output steps of stepper motor step (angle displacement) is always equal to the input number of pulses, so it can work as the open-loop position system. In terms of incremental motion, stepper motor can be used as a start-stop motion controller that is capable of rapid acceleration, deceleration and stop.

DC servo motor driver; DC servo motor has characteristics of good speed regulation characteristic, large starting torque, relatively large power and fast response, and the control technology is mature. But it has complex structure, large volume, high cost, and needs peripheral conversion circuit to realize digital control with microcomputer cooperating with outer switching circuit. If using DC servo motor, brush discharge effect on actual work should be considered.

AC servo motor drive; AC servo motor structure is relatively simple with small volume, reliable operation, convenient operation and maintenance, and the price is cheaper than DC servo motor but higher than step motor. With the development of turn off thyristor's decision, high power thyristo, GTR field-effect tube MOSFET and other electronic devices, pulse width adjusting technology and computer control technology, AC servo motor can be comparable to DC motor in speed control performance.

Hydraulic servo motor drive; Hydraulic servo motor has higher power/volume ratio, smooth movement, high positioning accuracy, and the load capacity is bigger, that can seize the heavy load without sliding. From the size, weight and required driving power several key technical considerations, it can yet be regarded as a suitable alternative.

\section{Conclusion}

Based on the current research status and development trend of industrial robots, the paper studies structure design, kinematics analysis and trajectory planning, work space analysis and motion simulation of a three degrees of freedom joint robot arms. The results are concluded mainly in the following several aspects:

First of all, according to the research status at home and abroad, combining with the characteristics of serial robot structure and the actual requirements, the design scheme of a series three degrees of freedom test robot is confirmed, realizing the structure design and drive solutions design, the results showed that the identified scheme is feasible.

The paper performs robot kinematics analysis, and the positive and inverse solution of the equation was deduced to solv, obtaining the jacobian matrix of the robot, making the solution interface of the kinematics positive and inverse solutions. The linkage velocity analysis and trajectory planning is prepared. 


\section{References}

[1] Kuroki Y, Ishida T, Yamaguchi J. Motion controller and motion control method for legged walking robot, and robot apparatus: US, US 7031806 B2[P]. 2006.

[2] Abdallah M E, Platt R, Wampler Ii C W. Hierarchical robot control system and method for controlling select degrees of freedom of an object using multiple manipulators: US, US8483882[P]. 2013.

[3] Nagasaka K. Control system, control method, and robot apparatus: US, US8463433 [P]. 2013.

[4] Hong Y S, Lee C W. Foot system for jointed leg type walking robot: US, US5758734 [P]. 1998.

[5] Nagasaka K. Actuator control device, actuator control method, actuator, robot apparatus, and computer program: US, US 8340822 B2 [P]. 2012.

[6] Parenti-Castelli V, Schiehlen W O. ROMANSY 18 - Robot Design, Dynamics and Control proceedings of the 18th CISM-IFToMM symposium[M]. Springer, 2010.

[7] Qiu J H, Jiang G. Waist design and motion simulation for pneumatic type humanoid robot [J]. Machinery Design \& Manufacture, 2012.

[8] Profio U D, Fujita M, Takagi T, et al. Robot apparatus, and behavior controlling method for robot apparatus: US, US 7103447 B2[P]. 2006.

[9] Mori N, Yamaguchi J. Legged walking robot and motion control method therefor: US, US7278501 [P]. 2007.

[10]Nakamura Y, Okada M, Shinohara T. A double spherical robot joint: EP, EP1334807 [P]. 2003.

[11]Saijo H, Kuroki Y. Humanoid robot communicating with body language: EP, EP1136193 [P]. 2001.

[12]Kobayashi K. Singing voice synthesizing method and apparatus, program, recording medium and robot apparatus: US, US7173178 [P]. 2007. 